\title{
Teores de clorofila em cultivares de tomateiro submetidas a aplicações foliares de magnésio ${ }^{1}$
}

\author{
Átila Francisco Mógor ${ }^{2}$, Thamiris Barbizan², Volnei Pauletti ${ }^{3}$, Juliana de Oliveira ${ }^{2}$, Marcelle Michelotti Bettoni ${ }^{2}$
}

\begin{abstract}
Chlorophyll contents in tomato cultivars under magnesium foliar applications

Although the foliar nutrients application is well disseminated in horticulture, the technology is still controversial, since the products efficiency is related to the characteristics of the formulations, especially the sources of nutrients used. Thus, this study aimed at identifying changes in chlorophyll contents, by using the colorimetric method and a chlorophyll meter, as well as in the $\mathrm{Mg}$ content in tomato plant leaves, as related to foliar applications of solutions containing $\mathrm{Mg}(\mathrm{OH})_{2}$ or $\mathrm{MgCl}_{2}$. Two tomato cultivars were subjected to fertigation with low $\mathrm{Mg}$ addition. The data from the chlorophyll meter were corroborated by the colorimetric method, indicating that the solutions containing $1.0 \mathrm{~mL} \mathrm{~L}^{-1}$ and $2.0 \mathrm{~mL} \mathrm{~L}^{-1}$ of a suspension concentrate (300 $\mathrm{g} \mathrm{L}^{-1}$ of $\mathrm{Mg}$, as $\mathrm{Mg}(\mathrm{OH})_{2}$ ) and the solution containing $3.75 \mathrm{~mL} \mathrm{~L}^{-1}$ of $\mathrm{MgCl}_{2}$ were effective in increasing the chlorophyll contents, resulting in the $\mathrm{Mg}$ absorption and metabolization. The solution containing $7.5 \mathrm{~mL} \mathrm{~L}^{-1}$ of $\mathrm{MgCl}_{2}$ caused fitotoxicity and reduced the chlorophyll contents, although promoting a higher $\mathrm{Mg}$ content in leaves.
\end{abstract}

KEY-WORDS: Solanum lycopersicum L.; magnesium hydroxide; magnesium chloride; portable chlorophyll-meter.

\section{INTRODUÇÃO}

Algumas técnicas de produção do tomateiro (Solanum lycopersicum L.), como a fertirrigação, adubação foliar, cobertura do solo e o cultivo em ambiente protegido, influenciam, positivamente, na absorção de nutrientes (Fayad et al. 2002) e podem alterar o estado nutricional das plantas, com reflexos, por exemplo, sobre os teores de clorofila nas folhas.

Por serem constituintes da molécula de clorofila, o $\mathrm{N}$ e o $\mathrm{Mg}$ são bastante estudados em plantas cultivadas, como, por exemplo, o tomateiro (Silva

\section{RESUMO}

Apesar de a aplicação de nutrientes via foliar ser bem disseminada na horticultura, a tecnologia ainda é controversa, pois a eficiência dos produtos está relacionada às características das formulações, especialmente quanto às fontes de nutrientes utilizadas. Assim, buscou-se, neste trabalho, identificar alterações nos teores de clorofila, utilizando o método colorimétrico e o método indireto por clorofilômetro, bem como alterações no teor de $\mathrm{Mg}$ nas folhas de tomateiro, em função de aplicações foliares de soluções contendo $\mathrm{Mg}(\mathrm{OH})_{2}$ ou $\mathrm{MgCl}_{2}$. Foram utilizadas duas cultivares, submetidas a fertirrigações com baixa adição de $\mathrm{Mg}$. Os valores das leituras com clorofilômetro foram corroborados pelo método colorimétrico, indicando que as soluções contendo $1,0 \mathrm{~mL} \mathrm{~L}^{-1}$ e 2,0 $\mathrm{mL} \mathrm{L}^{-1}$ de uma suspensão concentrada $\left(300 \mathrm{~g} \mathrm{~L}^{-1}\right.$ de $\mathrm{Mg}$, na forma de $\mathrm{Mg}(\mathrm{OH})_{2}$ ) e a solução contendo 3,75 $\mathrm{mL} \mathrm{L}^{-1}$ de $\mathrm{MgCl}_{2}$ foram eficientes em promover aumentos nos teores de clorofila, indicando a absorção e metabolização do Mg. Já a solução contendo $7,5 \mathrm{~mL} \mathrm{~L}{ }^{-1}$ de $\mathrm{MgCl}_{2}$ causou fitotoxidez, reduzindo os teores de clorofila, mas promovendo maior teor de $\mathrm{Mg}$ nas folhas.

PALAVRAS-CHAVE: Solanum lycopersicum L.; hidróxido de magnésio; cloreto de magnésio; clorofilômetro portátil.

et al. 2003). Desta forma, determinações periódicas dos teores de clorofila nas folhas podem auxiliar no manejo nutricional do tomateiro, em relação ao $\mathrm{N} \mathrm{e}$ Mg (Guimarães et al. 1999).

O método colorimétrico de determinação de clorofila (Lichtenthaler 1987) apresenta a desvantagem da coleta destrutiva do material vegetal. Já os medidores portáteis fornecem estimativas do conteúdo de clorofila das folhas diretamente no campo, sem a necessidade de destruição do material vegetal (Jesus \& Marenco 2008). Vários modelos de clorofilômetro vêm sendo utilizados para determinações

1. Trabalho recebido em out./2012 e aceito para publicação em set./2013 ( $\mathrm{n}^{\circ}$ registro: PAT 20592).

2. Universidade Federal do Paraná (UFPR), Departamento de Fitotecnia e Fitossanitarismo, Curitiba, PR, Brasil.

E-mails: atila.mogor@ufpr.br, tha barbizan@hotmail.com,juliever.ea@hotmail.com,m2bettoni@gmail.com.

3. Universidade Federal do Paraná (UFPR), Departamento de Solos e Engenharia Agrícola, Curitiba, PR, Brasil.

E-mail:vpauletti@ufpr.br. 
em pesquisas e programas de manejo nutricional (Arregui et al. 2006).

Apesar de a aplicação de nutrientes via foliar estar bem disseminada na horticultura, a tecnologia ainda é controversa, já que a eficiência dos produtos, em geral, está relacionada às características das formulações dos fertilizantes utilizados (Tanaka et al. 2003), especialmente quanto à natureza das fontes de nutrientes utilizadas.

Dessa forma, neste trabalho, buscou-se identificar e quantificar alterações nos teores de clorofila e $\mathrm{Mg}$, nas folhas de cultivares de tomateiro, em função de aplicações foliares crescentes de fontes de $\mathrm{Mg}$.

\section{MATERIAL E MÉTODOS}

O experimento foi conduzido em casa-de-vegetação do Departamento de Fitotecnia e Fitossanitarismo do Setor de Ciências Agrárias da Universidade Federal do Paraná (UFPR), em Curitiba (PR). Utilizaram-se as cultivares de tomateiro 'Granadeiro' e 'Dominador', dos grupos saladete e caqui, respectivamente (Agristar do Brasil 2011). A semeadura foi realizada em novembro de 2009, em bandejas de poliestireno expandido, com 200 células preenchidas com substrato agrícola comercial.

As mudas foram transferidas para vasos com volume de $10 \mathrm{~L}$, aos 28 dias após a emergência (DAE), quando apresentavam cinco folhas definitivas, com uma muda por vaso. O substrato utilizado foi composto por uma amostra de solo com as seguintes características químicas: $\mathrm{pH}\left(\mathrm{CaCl}_{2}\right)=5,1$; M.O. $=24,3 \mathrm{~g} \mathrm{dm}^{-3} ; \mathrm{P}=61 \mathrm{~g} \mathrm{dm}^{-3} ; \mathrm{K}=2,1 \mathrm{cmol} \mathrm{dm}^{-3}$; $\mathrm{Ca}=4,2 \mathrm{cmol}_{\mathrm{c}} \mathrm{dm}^{-3} ; \mathrm{Mg}=0,8 \mathrm{cmol}_{\mathrm{c}} \mathrm{dm}^{-3} ; \mathrm{CTC}=$ $14,77 \mathrm{cmol}_{\mathrm{c}} \mathrm{dm}^{-3}$; e $\mathrm{V}=48 \%$. Os vasos foram dispostos sobre bancadas de grade metálica, de forma a evitar o contato com o piso da casa-de-vegetação, e as plantas foram tutoradas e conduzidas com uma única haste. Após a emissão da terceira inflorescência, as plantas foram submetidas a poda apical, mantendo-se duas folhas emitidas imediatamente acima desta.

O turno de irrigação foi variável, de acordo com o nível de umidade do solo, identificado por vacuômetro, e as lâminas aplicadas visaram a manter a umidade do solo em torno de $90 \%$ da capacidade de campo.

O solo de cada vaso recebeu adubações em cobertura, semanalmente, por meio da aplicação de $100 \mathrm{~mL}$ de uma solução nutritiva completa, com baixo teor de $\mathrm{Mg}$ e condutividade elétrica de
$1,8 \mathrm{mS} \mathrm{cm}{ }^{-1}$, com as seguintes concentrações de nutrientes: $\mathrm{N}=100,0 \mathrm{mg} \mathrm{L}^{-1} ; \mathrm{P}=120,0 \mathrm{mg} \mathrm{L}^{-1} ; \mathrm{K}=$ $120,0 \mathrm{mg} \mathrm{L}^{-1} ; \mathrm{Ca}=85,0 \mathrm{mg} \mathrm{L}^{-1} ; \mathrm{Mg}=0,11 \mathrm{mg} \mathrm{L}^{-1} ; \mathrm{S}=$ $3,7 \mathrm{mg} \mathrm{L}^{-1} ; \mathrm{Fe}=0,30 \mathrm{mg} \mathrm{L}^{-1} ; \mathrm{B}=0,25 \mathrm{mg} \mathrm{L}^{-1} ; \mathrm{Mn}=$ $0,29 \mathrm{mg} \mathrm{L}^{-1} ; \mathrm{Zn}=0,38 \mathrm{mg} \mathrm{L}^{-1} ; \mathrm{Cu}=0,05 \mathrm{mg} \mathrm{L}^{-1} ; \mathrm{e}$ $\mathrm{Mo}=0,005 \mathrm{mg} \mathrm{L}^{-1}$.

Os tratamentos consistiram da aplicação foliar semanal de duas diferentes fontes de $\mathrm{Mg}$ (hidróxido de $\mathrm{Mg}$ e cloreto de $\mathrm{Mg}$ ), em duas diferentes doses de cada uma das fontes: $\mathrm{T} 1=1,0 \mathrm{~mL} \mathrm{~L}^{-1}$ de $\mathrm{Mg}(\mathrm{OH})_{2}$; $\mathrm{T} 2=2,0 \mathrm{~mL} \mathrm{~L}^{-1}$ de $\mathrm{Mg}(\mathrm{OH})_{2} ; \mathrm{T} 3=3,75 \mathrm{~mL} \mathrm{~L}^{-1}$ de $\mathrm{MgCl}_{2}$; e $\mathrm{T} 4=7,5 \mathrm{~mL} \mathrm{~L}^{-1}$ de $\mathrm{MgCl}_{2}$. As diferentes doses foram estabelecidas para uniformizar a concentração de $\mathrm{Mg}$ nas soluções aplicadas às folhas das plantas, sendo T1 e T3 correspondentes a $0,3 \mathrm{~g} \mathrm{~L}^{-1} \mathrm{de}$ $\mathrm{Mg}$, na solução, enquanto T2 e T4 corresponderam a $0,6 \mathrm{~g} \mathrm{~L}^{-1} \mathrm{de} \mathrm{Mg}$. Os tratamentos foram comparados entre si e com uma testemunha pulverizada com água destilada e deionizada, sendo distribuídos em arranjo inteiramente casualizado, em esquema fatorial $5 \times 2$ (tratamentos x cultivares), com quatro repetições, cada uma delas composta por quatro plantas.

$\mathrm{O}$ produto utilizado como fonte de $\mathrm{Mg}(\mathrm{OH})_{2}$ continha, em sua formulação, $69 \mathrm{~g} \mathrm{~L}^{-1}$ de $\mathrm{N}$, o que resultou em concentração de $138 \mathrm{mg} \mathrm{L}^{-1}$ de $\mathrm{N}$, no tratamento formulado com a maior dose de $\mathrm{Mg}(\mathrm{OH})_{2}$ $\left(2,0 \mathrm{~mL} \mathrm{~L}^{-1}\right)$. Por meio da adição de ureia, obteve-se a mesma concentração de $\mathrm{N}$ nos dois tratamentos formulados com $\mathrm{MgCl}_{2}$, na testemunha e no tratamento formulado com 1,0 mL L-1 $\mathrm{Mg}(\mathrm{OH})_{2}$. Também, acrescentou-se às misturas um adjuvante (éster metílico de óleo de soja) a $0,5 \%$, para melhorar o espalhamento da solução sobre as folhas.

As aplicações foliares tiveram início aos quinze dias após o transplantio das mudas (15 DAT), utilizando-se pulverizador pressurizado por $\mathrm{CO}_{2}, \mathrm{com}$ pressão constante de $3,16 \mathrm{kgf} \mathrm{cm}^{-2}$, o que possibilitou cobertura uniforme das plantas, evitando-se o escorrimento.

O teor relativo de clorofila nas folhas foi determinado utilizando-se clorofilômetro manual N-Tester ${ }^{\circledR}$ (Yara 2000), com funcionamento similar ao do clorofilômetro SPAD $502^{\circledR}$, que possui diodos que emitem radiação em $650 \mathrm{~nm}$ (luz vermelha) e $940 \mathrm{~nm}$ (radiação infravermelha). Durante a mensuração, a luz passa pela folha e é recebida por um fotodiodo de silício, sendo convertida, primeiramente, em sinais elétricos analógi$\cos$ e, depois, em sinais digitais. Estes sinais passam por um microprocessador que calcula valores proporcionais aos do teor de clorofila presente na folha (Minolta 1989). 
As leituras foram realizadas semanalmente, entre 18 DAT e 81 DAT, totalizando 9 avaliações, efetuadas três dias após a aplicação dos tratamentos. Para maior precisão, o N-Tester ${ }^{\circledR}$ apresenta, automaticamente, o valor médio de trinta leituras, sendo que os valores obtidos representam as médias das leituras de três folhas.

Assim, dos 18 DAT aos 46 DAT, procederam-se às leituras nas três folhas imediatamente abaixo do primeiro racimo e, dos 53 DAT aos 81 DAT, nas três folhas abaixo do segundo racimo. Aos 46 DAT, procedeu-se à retirada das folhas abaixo do primeiro racimo, para a determinação do teor de clorofila, pelo método colorimétrico descrito por Lichtenthaler (1987), bem como à determinação do teor de $\mathrm{Mg}$ na matéria seca do tecido vegetal (previamente lavado em água deionizada), segundo metodologia descrita por Malavolta et al. (1997). Adotou-se o mesmo procedimento aos 81 DAT, com a retirada das folhas abaixo do segundo racimo. Neste momento, procedeu-se à colheita dos frutos, sendo finalizado o experimento.

Os valores obtidos foram submetidos à análise de variância, sendo as médias comparadas pelo teste Tukey, a 5\%, utilizando-se o aplicativo Assistat 7.5.

\section{RESULTADOS E DISCUSSÃO}

As leituras dos teores relativos de clorofila (TRC) das folhas abaixo da primeira ráquis, aos 18 DAT, refletiram os baixos teores de $\mathrm{Mg}$ do solo, com a testemunha apresentando o menor valor médio, enquanto as plantas tratadas apresentaram valores superiores, porém, sem distinção entres os tratamentos de fontes e doses de Mg. Entre as cultivares, a 'Dominador' apresentou maior teor relativo de clorofila nas folhas, aos 18 DAT e 36 DAT, enquanto interações entre tratamentos e cultivares ocorreram entre 25 DAT e 32 DAT (Tabela 1).

O papel do Mg na síntese de clorofila é bem descrito na literatura (Larkin et al. 2003, Streit et al. 2005, Taiz \& Zaiger 2006) e a sua disponibilidade afeta o teor de clorofila nas folhas (Marschner 1995), como observado aos 18 DAT (Tabela 1), com leituras aos três dias após a primeira aplicação dos tratamentos, indicando que estes alteraram o TRC, ou seja, neste período, o Mg contido nas soluções foi absorvido pelas folhas e influenciou na síntese de clorofila.

As leituras do TRC, entre 18 DAT e 46 DAT, foram feitas nas três folhas abaixo do primeiro

Tabela 1. Teores relativos de clorofila das folhas de duas cultivares de tomateiro, localizadas abaixo da primeira ráquis, quantificados com clorofilômetro portátil N-Tester ${ }^{\circledR}$, entre 18 e 46 dias após o transplantio (DAT), em função de fontes e doses de Mg (Curitiba, PR, 2012).

\begin{tabular}{|c|c|c|c|c|c|c|c|}
\hline \multirow[b]{2}{*}{$\begin{array}{l}\text { Período de } \\
\text { avaliação }\end{array}$} & \multicolumn{7}{|c|}{ Tratamento } \\
\hline & Cultivar & $\begin{array}{l}\text { Controle } \\
\text { (água) }\end{array}$ & $\begin{array}{l}1,00 \mathrm{~mL} \mathrm{L-}{ }^{1} \\
\text { de } \mathrm{Mg}(\mathrm{OH})_{2}\end{array}$ & $\begin{array}{c}3,75 \mathrm{~mL} \mathrm{~L}^{-1} \\
\text { de } \mathrm{MgCl}_{2}\end{array}$ & $\begin{array}{l}2,00 \mathrm{~mL} \mathrm{~L}^{-1} \\
\text { de } \mathrm{Mg}(\mathrm{OH})_{2}\end{array}$ & $\begin{array}{l}\text { 7,5 mL L-1 } \\
\text { de } \mathrm{MgCl}_{2}\end{array}$ & Média \\
\hline & & \multicolumn{6}{|c|}{ - Teor relativo de clorofila } \\
\hline \multirow{4}{*}{$18 \mathrm{DAT}$} & Granadeiro & 401,5 & 459,0 & 478,2 & 509,0 & 452,2 & $460,0 \mathrm{~B}$ \\
\hline & Dominador & 443,0 & 526,7 & 540,5 & 483,0 & 502,0 & $499,0 \mathrm{~A}$ \\
\hline & Média & $422,2 b^{*}$ & $492,8 \mathrm{a}$ & $509,3 \mathrm{a}$ & $496,0 \mathrm{a}$ & $477,1 \mathrm{ab}$ & \\
\hline & $\mathrm{CV}$ & & & $8,72 \%$ & & & \\
\hline \multirow{3}{*}{$25 \mathrm{DAT}$} & Granadeiro & $464,7 \mathrm{bB}$ & $610,0 \mathrm{aA}$ & $660,7 \mathrm{aA}$ & $612,2 \mathrm{aA}$ & $507,5 \mathrm{bB}$ & \\
\hline & Dominador & $523,5 \mathrm{aA}$ & $656,7 \mathrm{aA}$ & $666,7 \mathrm{aA}$ & $647,2 \mathrm{aA}$ & $652,0 \mathrm{aA}$ & \\
\hline & $\mathrm{CV}$ & & & $6,51 \%$ & & & \\
\hline \multirow{3}{*}{32 DAT } & Granadeiro & $469,2 \mathrm{cB}$ & $615,5 \mathrm{aA}$ & $665,7 \mathrm{aA}$ & $615,0 \mathrm{bA}$ & $665,7 \mathrm{aA}$ & \\
\hline & Dominador & $541,7 \mathrm{bA}$ & $642,2 \mathrm{aA}$ & $662,5 \mathrm{aA}$ & $662,5 \mathrm{aA}$ & $662,5 \mathrm{aA}$ & \\
\hline & $\mathrm{CV}$ & & & $5,19 \%$ & & & \\
\hline \multirow{4}{*}{39 DAT } & Granadeiro & 484,5 & 589,2 & 639,2 & 599,7 & 465,5 & $555,6 \mathrm{~B}$ \\
\hline & Dominador & 568,0 & 623,0 & 644,7 & 664,5 & 550,7 & $610,2 \mathrm{~A}$ \\
\hline & Média & $526,2 \mathrm{~b}$ & 606,1 a & $642,0 \mathrm{a}$ & $632,1 \mathrm{a}$ & $508,1 \mathrm{~b}$ & \\
\hline & $\mathrm{CV}$ & & & $6,31 \%$ & & & \\
\hline \multirow{4}{*}{46 DAT } & Granadeiro & 449,7 & 494,2 & 531,7 & 508,5 & 425,7 & $482,0 \mathrm{~A}$ \\
\hline & Dominador & 504,5 & 514,7 & 535,2 & 545,0 & 433,0 & $506,5 \mathrm{~A}$ \\
\hline & Média & $477,1 \mathrm{ab}$ & 504,5 a & $533,5 \mathrm{a}$ & 526,7 a & $429,3 \mathrm{~b}$ & \\
\hline & $\mathrm{CV}$ & & & $9,31 \%$ & & & \\
\hline
\end{tabular}

* Valores seguidos da mesma letra minúscula, na linha, e maiúscula, na coluna, não diferem entre si, pelo Teste Tukey ( $\mathrm{p}<0,05)$. 
racimo. Neste período, a testemunha apresentou, em geral, os menores valores, contrastando com os demais tratamentos, que refletiram o efeito das aplicações foliares de $\mathrm{Mg}$, apresentando maior TRC, com as seguintes ressalvas: o tratamento com $7,5 \mathrm{~mL} \mathrm{~L}^{-1}$ de $\mathrm{MgCl}_{2}$ apresentou valor igual ao da testemunha, aos 18 DAT e 39 DAT, comparando-se os valores médios entre as duas cultivares, e aos 25 DAT, na cultivar 'Granadeiro'. Já aos 46 DAT, a testemunha apresentou valores próximos aos dos demais tratamentos, enquanto o tratamento com 7,5 $\mathrm{mL} \mathrm{L}^{-1}$ de $\mathrm{MgCl}_{2}$ apresentou TRC inferior, quando comparado à menor dose de $\mathrm{MgCl}_{2}$ e a ambas as doses de $\mathrm{Mg}(\mathrm{OH})_{2}$ (Tabela 1).

Essa observação indica fitotoxidez causada pela maior concentração de $\mathrm{MgCl}_{2}$ na solução de pulverização $\left(7,5 \mathrm{~mL} \mathrm{~L}^{-1}\right)$, já que não se verificou redução do TRC na metade da concentração de $\mathrm{MgCl}_{2}$ $\left(3,5 \mathrm{~mL} \mathrm{~L}^{-1}\right)$. Este efeito, provavelmente, se deve ao $\mathrm{Cl}$ e não ao $\mathrm{Mg}$, pois a solução contendo $2,0 \mathrm{~mL} \mathrm{~L}^{-1}$ da suspensão com $300 \mathrm{~g} \mathrm{~L}^{-1}$ de $\mathrm{Mg}$, na forma de $\mathrm{Mg}(\mathrm{OH})_{2}$, não apresentou fitotoxidez, tendo a mesma concentração de $\operatorname{Mg}\left(0,6 \mathrm{~g} \mathrm{~L}^{-1}\right)$.

Segundo Tavakkoli et al. (2010), o acúmulo de íons $\mathrm{Cl}$ no tecido vegetal pode alterar o potencial osmótico, afetando a permeabilidade das membranas celulares e promovendo a degradação da Chl, efeito que pode ser variável entre diferentes cultivares. Devido à sua alta mobilidade no floema, o $\mathrm{Mg}$ é facilmente redistribuído das folhas mais velhas para as mais jovens, em condições de baixa disponibilidade nas raízes das plantas (Marschner 1995), justificando a redução do TRC aos 46 DAT (Tabela 1). É necessário lembrar que os valores, neste período, são resultado das médias das leituras nas três folhas abaixo da primeira ráquis. Assim, os valores médios do TRC obtidos nas três folhas abaixo da segunda ráquis, aos $53 \mathrm{DAT}$ (703 e 623, respectivamente nas cultivares 'Granadeiro' e 'Dominador') (Tabela 2), foram superiores aos observados na leitura anterior, com TRC próximo a 500, para ambas as cultivares (Tabela 1), indicando a redistribuição do $\mathrm{Mg}$.

$\mathrm{Na}$ Tabela 2, encontram-se os teores relativos de clorofila identificados com clorofilômetro portátil, entre 53 DAT e 81 DAT, sendo observadas interações entre as fontes de $\mathrm{Mg}$ e as cultivares para todas as datas, exceto aos 53 DAT, quando foram observados efeitos isolados dos fatores.

$\mathrm{O}$ aumento do TRC na testemunha, entre 18 DAT e 46 DAT (Tabela 1), pode estar relacionado ao efeito do teor, mesmo que baixo, de $\mathrm{Mg}$ no solo e na solução nutritiva aplicada ao solo, associado ao pequeno conteúdo de $\mathrm{N}$ na solução aplicada às folhas, embora tenha permanecido com valores inferiores

Tabela 2. Teores relativos de clorofila das folhas de duas cultivares de tomateiro localizadas abaixo da primeira ráquis, quantificados com clorofilômetro portátil N-Tester ${ }^{\circledR}$, entre 53 e 81 dias após o transplantio (DAT), em função de fontes e doses de Mg (Curitiba, PR, 2012).

\begin{tabular}{|c|c|c|c|c|c|c|c|}
\hline \multirow{2}{*}{$\begin{array}{l}\text { Período de } \\
\text { avaliação }\end{array}$} & \multirow[b]{2}{*}{ Cultivar } & \multicolumn{5}{|c|}{ Tratamento } & \multirow[b]{2}{*}{ Média } \\
\hline & & $\begin{array}{l}\text { Controle } \\
\text { (água) }\end{array}$ & $\begin{array}{c}1,00 \mathrm{~mL} \mathrm{~L}^{-1} \\
\text { de } \mathrm{Mg}(\mathrm{OH})_{2}\end{array}$ & $\begin{array}{c}3,75 \mathrm{~mL} \mathrm{~L}^{-1} \\
\text { de } \mathrm{MgCl}_{2}\end{array}$ & $\begin{array}{l}2,00 \mathrm{~mL} \mathrm{~L}^{1} \\
\text { de } \mathrm{Mg}(\mathrm{OH})_{2}\end{array}$ & $\begin{array}{l}\text { 7,5 mL L- }{ }^{-1} \\
\text { de } \mathrm{MgCl}_{2}\end{array}$ & \\
\hline & & & & Teor relativo & clorofila - & & \\
\hline \multirow{4}{*}{53 DAT } & Granadeiro & 725,2 & 726,5 & 685,5 & 716,7 & 663,7 & $703,5 \mathrm{~A}$ \\
\hline & Dominador & 619,5 & 654,5 & 645,2 & 641,5 & 577,5 & $627,6 \mathrm{~B}$ \\
\hline & Média & $672,3 \mathrm{a}^{*}$ & $690,5 \mathrm{a}$ & $665,3 \mathrm{ab}$ & $679,1 \mathrm{a}$ & $620,6 \mathrm{~b}$ & \\
\hline & $\mathrm{CV}$ & & & $5,29 \%$ & & & \\
\hline \multirow{3}{*}{60 DAT } & Granadeiro & $714,0 \mathrm{aA}$ & $714,2 \mathrm{aA}$ & $697,5 \mathrm{aA}$ & $725,2 \mathrm{aA}$ & $532,0 \mathrm{bC}$ & \\
\hline & Dominador & $613,7 \mathrm{bBC}$ & $677,0 \mathrm{aA}$ & $685,5 \mathrm{aA}$ & $653,5 \mathrm{bAB}$ & $588,5 \mathrm{aC}$ & \\
\hline & $\mathrm{CV}$ & & & $4,35 \%$ & & & \\
\hline \multirow{3}{*}{67 DAT } & Granadeiro & $709,5 \mathrm{aA}$ & $728,7 \mathrm{aA}$ & $727,0 \mathrm{aA}$ & $722,0 \mathrm{aA}$ & $656,0 \mathrm{aB}$ & \\
\hline & Dominador & $599,7 \mathrm{bB}$ & $715,0 \mathrm{aA}$ & $755,5 \mathrm{aA}$ & $725,2 \mathrm{aA}$ & $557,2 \mathrm{bB}$ & \\
\hline & $\mathrm{CV}$ & & & $3,29 \%$ & & & \\
\hline \multirow{3}{*}{74 DAT } & Granadeiro & $671,2 \mathrm{aA}$ & $671,5 \mathrm{aA}$ & $671,5 \mathrm{aA}$ & $660,5 \mathrm{aAB}$ & $599,5 \mathrm{aB}$ & \\
\hline & Dominador & $609,7 \mathrm{bB}$ & $657,2 \mathrm{aAB}$ & $690,2 \mathrm{aA}$ & $673,7 \mathrm{aAB}$ & $521,0 \mathrm{bC}$ & \\
\hline & $\mathrm{CV}$ & & & $5,25 \%$ & & & \\
\hline \multirow{3}{*}{81 DAT } & Granadeiro & $410,7 \mathrm{aB}$ & $582,0 \mathrm{aA}$ & $536,5 \mathrm{aA}$ & $559,2 \mathrm{aA}$ & $333,0 \mathrm{bC}$ & \\
\hline & Dominador & $413,0 \mathrm{aB}$ & $558,7 \mathrm{aA}$ & $546,7 \mathrm{aA}$ & $521,7 \mathrm{bA}$ & $392,0 \mathrm{aB}$ & \\
\hline & $\mathrm{CV}$ & & & $6,96 \%$ & & & \\
\hline
\end{tabular}

* Valores seguidos da mesma letra minúscula, na linha, e maiúscula, na coluna, não diferem entre si, pelo Teste Tukey ( $\mathrm{p}<0,05)$. 
aos dos demais tratamentos. Isto indica que a metodologia adotada possibilitou isolar o efeito do $\mathrm{Mg}$ contido nas soluções, inclusive do efeito fitotóxico da solução contendo 7,5 $\mathrm{mL} \mathrm{L}^{-1} \mathrm{de} \mathrm{MgCl}_{2}$, que, após 39 DAT, apresentou maior redução do TRC, nas duas cultivares (Tabela 1).

Os teores de clorofila extraída de folhas coletadas aos 46 DAT corroboram os valores de TRC obtidos com o N-Tester ${ }^{\circledR}$, com as maiores concentrações de $\mathrm{Chl}$ encontradas no tecido das folhas submetidas às aplicações foliares com soluções contendo $\mathrm{Mg}(\mathrm{OH})_{2}$ e 3,75 $\mathrm{mL} \mathrm{L}^{-1}$ de $\mathrm{MgCl}_{2}$, indicando a absorção e metabolização do $\mathrm{Mg}$, enquanto a testemunha e o tratamento com $7,5 \mathrm{~mL} \mathrm{~L}^{-1} \mathrm{de}_{\mathrm{MgCl}_{2}}$ apresentaram os menores teores de Chl (Tabela 3 ). $\mathrm{Na}$ Tabela 3, encontram-se os teores de clorofila $\left(\mathrm{mg} \mathrm{kg}^{-1}\right)$ da massa das folhas, aos 46 DAT e 81 DAT, sendo observados apenas efeitos isolados dos fatores, sem interações.

Schuelter et al. (2003) verificaram alterações no teor de clorofila das folhas de plantas de tomate variando em função das cultivares, idade e posição das folhas coletadas, com redução dos teores ao longo do tempo e aumento dos teores nas folhas imediatamente acima das lidas em períodos anteriores, de forma similar ao encontrado no presente trabalho, com as variações do TRC nas cultivares ao longo do segundo período de leituras e redução dos valores a partir de 74 DAT (Tabela 2), repetindo o ocorrido aos 39 DAT, nas folhas abaixo do primeiro racimo (Tabela 1). Os autores também encontraram correspondência entre o teor de Chl e o TRC.

O TRC, nas folhas abaixo do segundo racimo, refletiu a redistribuição do $\mathrm{Mg}$, inclusive na teste- munha, e a fitotoxidez das plantas que receberam 7,5 $\mathrm{mL} \mathrm{L}^{-1} \mathrm{de} \mathrm{MgCl}_{2}$ aos 53 DAT, comportamento que se manteve até os 74 DAT, na cultivar 'Granadeiro'. $\mathrm{Na}$ 'Dominador', o TRC refletiu o efeito das aplicações foliares a partir de 60 DAT, com os maiores valores observados nos tratamentos contendo $\mathrm{Mg}(\mathrm{OH})_{2}$ e menor concentração de $\mathrm{MgCl}_{2}$, observação que foi comum às duas cultivares, aos 81 DAT (Tabela 2), no final do experimento.

As médias dos teores de Mg, nas folhas coletadas aos 46 DAT e 81 DAT, são apresentadas na Tabela 4, indicando que ocorreu interação entre tratamentos e cultivares. A cultivar 'Granadeiro' apresentou, em geral, maiores teores de $\mathrm{Mg}$ do que a 'Dominador', especialmente na ausência e nas menores doses de $\mathrm{Mg}$ aplicadas. Os teores variaram entre $5,1 \mathrm{~g} \mathrm{~kg}^{-1}$ e 9,6 $\mathrm{g} \mathrm{kg}^{-1}$, com exceção do tratamento com 7,5 mL L-1 de $\mathrm{MgCl}_{2}$, cujos valores variaram entre $11,2 \mathrm{~g} \mathrm{~kg}^{-1}$ e $12,3 \mathrm{~g} \mathrm{~kg}^{-1}$.

Outros autores encontraram teores variando de 6,5 $\mathrm{g} \mathrm{kg}^{-1}$ a 7,5 $\mathrm{g} \mathrm{kg}^{-1}$ de $\mathrm{Mg}$, na cv. Carmem (Pereira \& Mello 2002), do tipo caqui, ou de $4,5 \mathrm{~g} \mathrm{~kg}^{-1}$ a $6,4 \mathrm{~g} \mathrm{~kg}^{-1}$, em tomate tipo cereja (Fernandes et al. 2006), e de 6,5 $\mathrm{g} \mathrm{kg}^{-1}$ a 9,2 $\mathrm{g} \mathrm{kg}^{-1}$ de $\mathrm{Mg}$ (Fontes et al. 2004), também em cultivar do tipo caqui. No entanto, em nenhum dos trabalhos os autores relacionaram os teores foliares de $\mathrm{Mg}$ a sintomas de deficiência ou toxidez, ou à produção de frutos.

Ao se compararem os teores de Mg nas folhas da testemunha com os encontrados nos tratamentos com as menores doses aplicadas, especialmente na cultivar 'Dominador', bem como ao se compararem os teores nos tratamentos com as maiores doses (Tabela 4), seria razoável inferir que ocorreu maior eficiência da

Tabela 3. Teores de clorofila de folhas de duas cultivares de tomateiro, aos 46 e 81 dias após o transplantio (DAT), em função de fontes e doses de Mg aplicado via foliar (Curitiba, PR, 2012).

\begin{tabular}{|c|c|c|c|c|c|c|c|}
\hline \multirow[b]{2}{*}{$\begin{array}{l}\text { Período de } \\
\text { avaliação }\end{array}$} & \multirow[b]{2}{*}{ Cultivar } & \multicolumn{5}{|c|}{ Tratamento } & \multirow[b]{2}{*}{ Média } \\
\hline & & $\begin{array}{l}\text { Controle } \\
\text { (água) }\end{array}$ & $\begin{array}{l}1,00 \mathrm{~mL} \mathrm{~L}^{1} \\
\text { de } \mathrm{Mg}(\mathrm{OH})_{2}\end{array}$ & $\begin{array}{c}3,75 \mathrm{~mL} \mathrm{~L}^{-1} \\
\text { de } \mathrm{MgCl}_{2}\end{array}$ & $\begin{array}{l}2,00 \mathrm{~mL} \mathrm{~L}^{1} \\
\text { de } \mathrm{Mg}(\mathrm{OH})_{2}\end{array}$ & $\begin{array}{l}\text { 7,5 } \mathrm{mL} \mathrm{L}^{1} \\
\text { de } \mathrm{MgCl}_{2}\end{array}$ & \\
\hline \multirow{5}{*}{46 DAT } & & & & Teor de cloro & $a\left(m g k^{-1}\right)$ & & \\
\hline & Granadeiro & 308,1 & 420,2 & 443,2 & 511,0 & 312,9 & $399,1 \mathrm{~A}$ \\
\hline & Dominador & 307,1 & 483,6 & 483,6 & 456,3 & 270,2 & $418,0 \mathrm{~A}$ \\
\hline & Média & $307,6 b^{*}$ & $451,9 \mathrm{a}$ & $463,4 \mathrm{a}$ & $483,7 \mathrm{a}$ & $291,5 \mathrm{~b}$ & \\
\hline & $\mathrm{CV}$ & & & $13,22 \%$ & & & \\
\hline \multirow{4}{*}{81 DAT } & Granadeiro & 379,9 & 456,7 & 448,3 & 415,4 & 304,3 & $400,9 \mathrm{~A}$ \\
\hline & Dominador & 327,5 & 385,1 & 451,5 & 459,9 & 323,2 & $389,4 \mathrm{~A}$ \\
\hline & Média & $353,7 \mathrm{bc}$ & $420,9 \mathrm{ab}$ & $449,9 \mathrm{a}$ & $437,6 \mathrm{a}$ & $313,8 \mathrm{c}$ & \\
\hline & $\mathrm{CV}$ & & & $13,18 \%$ & & & \\
\hline
\end{tabular}

* Valores seguidos da mesma letra minúscula, na linha, e maiúscula, na coluna, não diferem entre si, pelo Teste Tukey (p < 0,05). 
Tabela 4. Médias dos teores de Mg da massa seca das folhas de duas cultivares de tomateiro, aos 46 e 81 dias após o transplantio (DAT), em função de fontes e doses de Mg aplicado via foliar (Curitiba, PR, 2012).

\begin{tabular}{|c|c|c|c|c|c|c|}
\hline \multirow{2}{*}{$\begin{array}{l}\text { Período de } \\
\text { avaliação }\end{array}$} & \multirow[b]{2}{*}{ Cultivar } & \multicolumn{5}{|c|}{ Tratamento } \\
\hline & & $\begin{array}{c}\text { Controle } \\
\text { (água) }\end{array}$ & $\begin{array}{l}1,00 \mathrm{~mL} \mathrm{~L}^{1}{ }^{1} \\
\text { de } \mathrm{Mg}(\mathrm{OH})_{2}\end{array}$ & $\begin{array}{c}3,75 \mathrm{~mL} \mathrm{L-}^{1} \\
\text { de } \mathrm{MgCl}_{2}\end{array}$ & $\begin{array}{l}2,00 \mathrm{~mL} \mathrm{~L}^{1} \\
\text { de } \mathrm{Mg}(\mathrm{OH})_{2}\end{array}$ & $\begin{array}{l}\text { 7,5 } \mathrm{mL} \mathrm{L}^{-1} \\
\text { de } \mathrm{MgCl}_{2}\end{array}$ \\
\hline & & & 1 & de $M g\left(g \mathrm{~kg}^{-1}\right.$ & +2 & \\
\hline \multirow{3}{*}{46 DAT } & Granadeiro & $7,9 \mathrm{bA}^{*}$ & $8,4 \mathrm{bA}$ & $9,4 \mathrm{abA}$ & $9,6 \mathrm{abA}$ & $11,2 \mathrm{aA}$ \\
\hline & Dominador & $5,1 \mathrm{cB}$ & $5,7 \mathrm{cB}$ & $7,4 \mathrm{bB}$ & $7,4 \mathrm{bB}$ & $12,3 \mathrm{aA}$ \\
\hline & $\mathrm{CV}$ & & & $15,7 \%$ & & \\
\hline \multirow{3}{*}{$81 \mathrm{DAT}$} & Granadeiro & $8,5 \mathrm{bA}$ & $8,1 \mathrm{bA}$ & $8,5 \mathrm{bA}$ & $8,9 \mathrm{bA}$ & $12,1 \mathrm{aA}$ \\
\hline & Dominador & $5,7 \mathrm{cB}$ & $6,5 \mathrm{cB}$ & $9,4 \mathrm{bA}$ & $7,7 \mathrm{bcA}$ & $12,2 \mathrm{aA}$ \\
\hline & $\mathrm{CV}$ & & & $12,0 \%$ & & \\
\hline
\end{tabular}

fonte $\mathrm{MgCl}_{2}$ no fornecimento de $\mathrm{Mg}$ para as plantas do tomateiro, em detrimento ao $\mathrm{Mg}(\mathrm{OH})_{2}$. Entretanto, os maiores teores de $\mathrm{Mg}$ nas folhas (Tabela 4) não refletiram em aumento dos teores de Chl (Tabela 3) e TRC (Tabelas 1 e 2). Assim, verificou-se que as concentrações de $\mathrm{Mg}$ nas folhas não expressaram, na totalidade, a eficiência dos tratamentos, já que a dose de 7,5 $\mathrm{mL} \mathrm{L}^{-1}$ de $\mathrm{MgCl}_{2}$ apresentou os maiores teores foliares de $\mathrm{Mg}$, aliados aos menores de $\mathrm{Chl}$ e menores valores de TRC, enquanto os tratamentos com $\mathrm{Mg}(\mathrm{OH})_{2}$ e $3,75 \mathrm{~mL} \mathrm{~L}^{-1}$ de $\mathrm{MgCl}_{2}$ apresentaram teores intermediários de $\mathrm{Mg}$, mas superiores aos de Chl (Tabela 3) e TRC (Tabelas 1 e 2).

Aos 81 DAT, procedeu-se à colheita dos frutos, cujos resultados não foram comparados entre as cultivares, devido ao fato de estas pertencerem a grupos diferentes, sendo 'Granadeiro' do grupo saladete, apresentando frutos do tipo oblongo, e 'Dominador' do grupo caqui, com frutos mais achatados (Agristar do Brasil 2011). Como em outros trabalhos citados (Pereira \& Mello 2002, Fontes et al. 2004, Fernandes et al. 2006), não foi verificado reflexo dos teores de $\mathrm{Mg}, \mathrm{Chl}$ e valores de TRC na produção.

\section{CONCLUSÃO}

A absorção foliar e a metabolização das soluções contendo a suspensão concentrada (flowable) de $\mathrm{Mg}(\mathrm{OH})_{2}$, nas duas concentrações testadas, e a solução contendo 3,75 mL L-1 de $\mathrm{MgCl}_{2}$ mostraram-se evidentes, por promoverem aumentos nos teores de clorofila. A solução contendo 7,5 mL L-1 de $\mathrm{MgCl}_{2}$ reduziu o teor de clorofila nas folhas, apesar de aumentar o conteúdo de $\mathrm{Mg}$, indicando fitotoxidez causada pelo $\mathrm{Cl}$.

\section{AGRADECIMENTOS}

À empresa Yara, por ceder os produtos utilizados nesta pesquisa.

\section{REFERÊNCIAS}

AGRISTAR DO BRASIL. Características de cultivares de tomate. São Paulo: Sementes Top Seed Premium, 2011.

ARREGUI, L. M. et al. Evaluation of chlorophyll meters as tools for $\mathrm{N}$ fertilization in winter wheat under humid Mediterranean conditions. European Journal of Agronomy, Rome, v. 24, n. 2, p. 140-148, 2006.

FAYAD, J. A. et al. Absorção de nutrientes pelo tomateiro cultivado sob condições de campo e de ambiente protegido. Horticultura Brasileira, Brasília, DF, v. 20, n. 1, p. 9094, 2002.

FERNANDES, C.; CORÁ, J. E.; BRAZ, L. T. Desempenho de substratos no cultivo do tomateiro do grupo cereja. Horticultura Brasileira, Brasília, DF, v. 24, n. 1, p. 4246, 2006.

FONTES, P. C. R. et al. Produção e qualidade do tomate produzido em substrato, no campo e em ambiente protegido. Horticultura Brasileira, Brasília, DF, v. 22, n. 1, p. 614-619, 2004.

GUIMARÃES, T. C. et al. Teores de clorofila determinados por medidor portátil e sua relação com formas de nitrogênio em folhas de tomateiro cultivado em dois tipos de solo. Bragantia, Campinas, v. 58, n. 1, p. 209-216, 1999.

JESUS, S. V.; MARENCO, R. A. O. O SPAD-502 como alternativa para a determinação dos teores de clorofila em espécies frutíferas. Acta Amazonica, Manaus, v. 38, n. 1, p. 815-818, 2008. 
LARKIN, R. M. et al. GUN4, a regulator of chlorophyll synthesis and intracellular signaling. Science, Washington, DC, v. 299, n. 5608, p. 902-906, 2003.

LICHTENTHALER, H. K. Chlorophylls and carotenoids: pigments of photosynthetic biomembranes. In: PACKER, L.; DOUCE, R. (Eds.). Methods in enzimology. London: Academic Press, 1987. p. 350-381.

MALAVOLTA, E.; VITTI, G. C.; OLIVEIRA, S. A. Avaliação do estado nutricional de plantas: princípios e aplicações. 2. ed. Piracicaba: Potafos, 1997.

MARSCHNER, H. Mineral nutrition of higher plants. 2. ed. San Diego: Academic Press, 1995.

MINOLTA. Radiometric Instruments Division. Manual for chlorophyll meter SPAD 502. Osaka: Minolta, 1989.

PEREIRA, H. S.; MELLO, S. C. Aplicações de fertilizantes foliares na nutrição e na produção do pimentão e do tomateiro. Horticultura Brasileira, Brasília, DF, v. 20, n. 1, p. 597-600, 2002.

SCHUELTER, A. R. et al. Avaliação dos níveis de clorofila em folhas de tomateiro da cultivar Santa Clara, do mutante 'firme' e do híbrido F1. Acta Scientiarum Agronomy, Maringá, v. 25, n. 1, p. 183-187, 2003.
SILVA, J. B. C. et al. Cultivo do tomate para industrialização. 2003. Disponível em: <http://www. cnph.embrapa.br/sistprod/tomate/def_nutricional.htm>. Acesso em: 23 mar. 2011.

STREIT, N. M. et al. As clorofilas. Ciência Rural, Santa Maria, v. 35, n. 1, p. 748-755, 2005.

TAIZ, L.; ZEIGER, E. Plant physiology. Sunderland: Sinauer Associates, 2006.

TANAKA, M. T. et al. Efeito da aplicação foliar de biofertilizantes, bioestimulantes e micronutrientes na cultura do tomateiro (Lycopersicon esculentum Mill.). Acta Scientiarum Agronomy, Maringá, v. 25, n. 1, p. 315321, 2003.

TAVAKKOLI, E. et al. Additive effects of $\mathrm{Na}^{+}$and $\mathrm{Cl}^{-}$ ions on barley growth under salinity stress. Journal of Experimental Botany, Lancaster, v. 62, n. 1, p. 2189-2203, 2010.

YARA. How to use the N-Tester. 2000. Disponível em: <http://adamchukpa.mcgill.ca/gpt_seminar/gpt_ present/0510.pdf $>$. Acesso em: 4 jun. 2013. 